\title{
New advanced technology in medicine: Bio electron's photons
}

\author{
Nick Kostovic* \\ Bio Technological Health Center, Inc., 3838 Carson Street, Suite 307, Torrance, CA 90503, Los Angeles, USA
}

Bio-electric current is converted from standard electricity, by using a bio-electron-laser. This energy format can deeply penetrate into any physical organ, including the brain with no risk. It is absolutely safe, none-lethal, and does not cause any electrical shocks.

This revolutionary technology using 120 Voltages of AC knowing the Human body's resistance to ground is could be $1,000 \mathrm{Ohms}$, produces the effect of approximately 12 Micro Amperes. By utilizing Nick's newly discovered Reverse Current we cancel magnetic from electricity and thereby prevent electromagnetic shock. Which means this K-BTE device produces 10,000 times less speed - strength of electrons then today's developed technology. Throughout the world today, the most advanced technology can only perform at the Mili Amperes frequency which is too high speed-strength of electrons, and for this reason it is considered electric shock therapy and is lethal. We are the only Center in the world who has successfully canceled magnetic from electromagnetic enabling us to perform at the unrivaled gentle frequency of Micro Amperes and Nano Amperes.

The Kostovic Bio-Technology Energetic Device has the ability to extract bio-electron photons from $\mathrm{H} 2 \mathrm{O}$ electric fluid. Creating $1 \mathrm{~A}=$ $1,000,000$ Micro Amperes. Using the power of standard 120 Voltages yet creating such a very low frequency of Micro Amperes we do not create any electric shock, it is NOT at all lethal. We can also bring the frequency down even smaller to the Nano amperes level with is $1 \mathrm{~A}=$ $1,000,000,000$ billionth Nano amperes and control them like tame sheep.

My Center discovered technology produces photons of bio electrons at this Micro amperes and Nano amperes level which is gentle yet precise and efficient in burning off oxidized proteins and dead cells while energizing the hibernating healthy cells within the vascular system and the injured organ. This advanced technological laser is capable of performing medical surgeries on the brain, heart, lungs, kidneys, all cancers in any physical organ with no harm to the healthy cells and of course with no negative side effects. He has been performing treatments for last 15 years in USA with more than 40,000 hours using these gentle Micro Amperes and Nano Amperes frequencies.

\section{The process}

Bio Electrons are extracted from $\mathrm{H} 20$, and enriched by an assortment of natural acids, linoleic acid, oleic acid, fig acids, etc. These bio electric photons deeply penetrate throughout any damaged or sick physical organ. Our process emits sheaves of enriched bio electrons of elementary biochemical electrical charges destroying and dispersing the unhealthy cells from fiber tissue while simultaneously nourishing and stimulating the healthy cells to multiply/divide. These mostly multi-chromatic gold or platinum-like sheaves of enriched bio electrons deeply penetrate into the fiber tissue of any physical tissue. All other lasers are solely monochromatic (single wavelength).

Using this bio electricity, we can wirelessly "burn off" and disperse dead cells/oxidized proteins from atrophied muscle tissue, bone tissue, cartilage tissue, malignant tumors with metastasis in the brain, breast or any other physical organ, with no harm to the healthy cells. These released amino acid enriched electrons of bio electricity also stimulate the growth of new daughter cells otherwise known as the "mitotic cell division phase". Resulting in the rebuilding of fiber cells in skin, muscle, bone and cartilage tissue within any physical organ.

Our proven technological discovery is the most efficient technique in the world in healing brain tumors. We can also stop the progression of Alzheimers and dementia and many times reverse it but without a doubt we can halt the progression which modern medicine readily admits can only slow down the decline.

We have MRI's and SCAT scans to substantiate our claims.

Today's standard technological advanced medical surgeries use lasers which can only perform at the speed/strength of Mili Amperes, which is: $1 \mathrm{~A}=1,000$ Mili Amperes.

Micro Amperes maximum strength output among the first energetic field and the second energetic field on the floor of K-BTE are 2 DC Micro Amperes to 15 DC Micro Amperes, with average of 8.5 Direct Current Micro Amperes.

This measured current is confirmed for the body's resistance from 1,000 to 100,000 Ohms or more, it doesn't matter dry or wet. The severity of any lethal shock is absolutely eliminated as these voltages can't produce a dangerous shock.

The technician's hands feel a slight increase due to a repelling / opposite of attracting/ tingling sensation, because it is not wires in output, and it is not electromagnetic. This is why this device is so revolutionary because it is connected in a special circuit by $\mathrm{DC} /$ direct current/, AC /alternating current/ and RC /reverse current/.

Today's scientific formula for electromagnetic current power it is:

Correspondence to: Nick Kostovic, Bio Technological Health Center, Inc. 3838 Carson Street, Suite 307, Torrance, CA 90503, Los Angeles, USA, Tel: +1-310-543-0309/+1-310-381-9105; Fax: +1-310-540-6946; E-mail: nick@ kbhealthcenter.com

Received: October 05, 2016; Accepted: October 20, 2016; Published: October 24,2016 
$\mathrm{I}=\mathrm{V}: \mathrm{R}$ ( $\mathrm{I}=$ current; $\mathrm{V}=$ Voltages and $\mathrm{R}=$ Human resistance to ground in Ohms)

Please understand that if the human body's resistance is 1,000 to $100,000 \mathrm{Ohm}$ and more, and using 120 Voltages which is 1.2 to 0,12 Mili Amperes or 12,000 decreasing to 1,200 Micro Amperes, then it's threshold impact, would result in electric shock and death. This is today's current official technological development in laser medicine. !!!

This revolutionary technology using 120 Voltages of AC knowing the Human body's resistance to ground is $1,000 \mathrm{Ohm}$ to 100,000 , yet produces the effect of about 12 Micro Amperes. By utilizing this newly discovered Reverse Current we prevent electromagnetic shock. Which means this K-BTE laser medical device is 10,000 times less in Micro Amperes strength than today's developed technology. So these penetrations on the surface of the skin are soft, not electric shock, it doesn't matter the human body's resistance is 1,000 , or 100,000 or $1,000,000$ it just doesn't matter anymore because we are producing such low Micro Amperes. We can even bring it down to the Nano amperes level which is 1 billionth of the amplitude.

So this worldwide standard scientific formula I=V:R doesn't apply to this newly developed technology, this formula is now history!

This combination of high Voltages with low Micro Amperes are capable to destroy and kill various Cancer, Viruses, Hepatitis Viruses, AIDS Viruses. We have no doubt when bio electric electrons are enriched with proper natural acids it can destroy and kill Ebola Viruses preventing future epidemics.

Copyright: ( 2016 Kostovic N. This is an open-access article distributed under the terms of the Creative Commons Attribution License, which permits unrestricted use, distribution, and reproduction in any medium, provided the original author and source are credited. 